\title{
Was folgt aus dem Brexit? Mögliche Szenarien differenzierter (Des-)Integration
}

\author{
Funda Tekin*
}

Am 23. Juni 2016 hat sich das britische Volk, wenn auch merklich knapp, mit 51,9 zu 48,1 Prozent für einen Austritt Großbritanniens aus der Europäischen Union, den sogenannten Brexit, ausgesprochen. Damit ist das eingetreten, womit niemand, erstaunlicherweise offensichtlich auch die Brexit-Befürworter nicht, ernsthaft gerechnet hatte. Das politische Europa rang am Tag danach um Contenance: Der Präsident des Europäischen Rates, Donald Tusk, sprach von einem „historischen Moment“ und warnte gleichzeitig vor „hysterischen Reaktionen“; ${ }^{1}$ Bundeskanzlerin Angela Merkel erklärte das Ergebnis zum „Einschnitt für den europäischen Einigungsprozess“ und riet an, Schlussfolgerungen mit „historischem Bewusstsein“ zu ziehen; ${ }^{2}$ und François Hollande sowie die Präsidenten von Europäischem Parlament, Europäischem Rat und der Kommission sowie die damals amtierende Ratspräsidentschaft sprachen von einer „schmerzhaften“ beziehungsweise „bedauerlichen“ Entscheidung, die jedoch respektiert werden müsse. ${ }^{3}$ Boris Johnson, ehemaliger Bürgermeister Londons und einer der Hauptakteure der Brexit-Kampagne, versuchte jedoch im Nachhinein diese Entscheidung zu relativieren, indem er herausstellte, dass Großbritannien immer ein Teil Europas gewesen ist und dieses auch bleiben wird. Darüber hinaus wird Johnson zufolge, der im neuen britischen Kabinett als Außenminister fungiert, der Zugang Großbritanniens zum europäischen Binnenmarkt bestehen bleiben. ${ }^{4}$

Diese Einschätzungen lassen bereits vermuten, dass das Ergebnis des Referendums Interpretationsspielräume eröffnet. Auch wenn Theresa May, die am 13. Juli 2016 das Premierministeramt von David Cameron übernommen hat, versichert, dass Brexit auch Brexit bedeutet, scheint es legitim zu erwarten, dass die politische Realität weniger eindeutig sein wird und verschiedene Szenarien als Ergebnis des Referendums denkbar sind.

Ziel dieses Beitrags ist es daher, die Bedeutung eines Austritts Großbritanniens aus der Europäischen Union im Lichte möglicher Szenarien und ihrer Implikationen sowie Konsequenzen sowohl für die künftige Europäische Union als auch für ihr Verhältnis zu Großbritannien herauszuarbeiten. Im Vorfeld des Referendums in Großbritannien wurde eine

* Dr. Funda Tekin, Centrum für Türkei und EU Studien, Universität zu Köln; Centre international de formation européenne, Nizza/Berlin; wissenschaftliche Beraterin des Instituts für Europäische Politik, Berlin.

1 Europäischer Rat, Der Präsident: Presseerklärung von Präsident Donald Tusk zum Ergebnis des Referendums im Vereinigten Königreich. Brüssel, 24. Juni 2016, Dok. 380/16.

2 Die Bundesregierung: Merkel zum Brexit. EU ist stark genug für die richtigen Antworten. Berlin, 24. Juni 2016, abrufbar unter: https://www.bundesregierung.de/Content/DE/Artikel/2016/06/2016-06-24-merkel-zu-bre xit-grossbritanien-stimmt-fuer-austritt-aus-eu.html;jsessionid=43EFB5786B44C3BF0FD94DC01768B863.s7t1 ?nn=694676 (letzter Zugriff: 18.7.2016).

3 Präsidentschaft der Französischen Republik: Déclaration à la suite du référendum britannique. Paris, 24. Juni 2016, abrufbar unter: http://www.elysee.fr/declarations/article/declaration-a-la-suite-du-referendum-britanniqu e/ (letzter Zugriff: 18.7.2016); Europäische Kommission: Gemeinsame Erklärung: Martin Schulz, Präsident des Europäischen Parlaments, Donald Tusk, Präsident des Europäischen Rates, Mark Rutte, Inhaber der Präsidentschaft des Rates, Jean-Claude Juncker, Präsident der Europäischen Kommission. Brüssel, 24. Juni 2016, STATEMENT/16/2329.

4 Siehe Boris Johnson: I cannot stress too much that Britain is part of Europe - and always will be, in: The Telegraph, 26.6.2016. 
Vielzahl von unterschiedlichen Szenarien für die Zukunft der Beziehungen zwischen der Europäischen Union und dem Vereinigten Königreich erarbeitet. ${ }^{5}$ Hier werden diese in drei aktuell noch immer realistisch erscheinende Hauptstränge eines (1) vollständigen Austritts, eines (2) Outs mit Opt-ins sowie eines (3) Ins mit Opt-outs gefasst. ${ }^{6}$

Für die Analyse rahmengebend sind konzeptionelle Überlegungen zur differenzierten Integration und Desintegration. In diesem Zusammenhang ist zu beachten, dass die theoretische Auseinandersetzung mit Möglichkeiten europäischer Desintegration noch sehr limitiert ist. ${ }^{7}$ Bisher war dieses Phänomen im europäischen Integrationsprozess trotz der multiplen und in den letzten Jahren akkumulierten Krisen noch nicht zu beobachten. Ein möglicher Brexit würde daher einen Präzedenzfall für einen Austritt aus der Europäischen Union und somit einer (differenzierten) Desintegration konstituieren. Die hier vorgelegte Analyse kann dazu beitragen, unterschiedliche Auslöser und Logiken sowohl differenzierter Integration als auch differenzierter Desintegration zu identifizieren. Ersteres gilt allgemein als ein Instrument für das Management von Heterogenität der EU-Mitgliedstaaten, um Integrationsfortschritt zu ermöglichen. Differenzierte Desintegration wäre hingegen als ein Scheitern der europäischen Integration an der Heterogenität der EU-Mitgliedstaaten zu interpretieren, welches zur Fragmentierung der Europäischen Union bis hin zu ihrer Auflösung führen kann.

Das grundlegende Argument dieses Beitrags ist, dass der Brexit einen Lackmustest für die europäische Integration darstellt, weil er je nach Szenario den bereits existierenden Trend differenzierter Integration unterschiedlich stark fördern würde. Dies könnte unter Umständen zu Desintegrationstendenzen der Europäischen Union führen.

\section{Großbritannien in der Europäischen Union: Eckdaten einer differenzierten Beziehung}

\section{Großbritannien und die Europäische Union - ein Verhältnis mit Stolpersteinen?}

Großbritannien trat 1973 - nach zwei aufgrund französischer Vetos misslungenen Versuchen in den 1960er Jahren - der Europäischen Wirtschaftsgemeinschaft bei. Somit kann bereits der Beginn der Beziehungen zwischen Großbritannien und der heutigen Europäischen Union als holprig bezeichnet werden. Diese sind seitdem speziell geblieben: Nur zwei Jahre nach dem Beitritt fand am 5. Juni 1975 in Großbritannien das erste Referendum zur Mitgliedschaft in der Europäischen Gemeinschaft statt. Damals führten ähnlich wie 2016 innenpolitische Umstände zur Ansetzung des Referendums. ${ }^{8}$ Das Ergebnis war jedoch ein anderes: 67 Prozent votierten für den Verbleib in der Europäischen Gemeinschaft. Großbritannien war immer und ist auch heute noch bestrebt, nationale Souveräni-

5 Siehe unter anderem Jean-Claude Piris: If the UK votes to leave. The seven alternatives to EU membership, Centre for European Reform: Policy Brief, Januar 2016; Heidi Marleen Kuhlmann: 5 Szenarien für Großbritannien. Brexit ist... was wir daraus machen, Jacques Delors Institut Berlin: Blog Post, 31. Mai 2016.

6 Opt-out ist der allgemeingültige Begriff für die primärrechtlich definierte Erlaubnis eines Staates, sich an einer gemeinsamen EU-Politik nicht beteiligen zu müssen. Opt-in bedeutet im Umkehrschluss das Recht, sich an EU-Politiken beteiligen zu können.

7 Siehe Henrik Scheller/Annegret Eppler: European Disintegration - non-existing Phenomenon or a Blind Spot of European Integration Research? Preliminary Thoughts for a Research Agenda, Institute for European Integration Research: Working Paper 2/2014; Douglas Webber: How likely is it that the European Union will disintegrate? A critical analysis of competing theoretical perspectives, in: European Journal of International Relations 2/2014, S. 341-365.

8 Siehe auch Guy de Jonquières: The UK Referendum - and the Future of the European Project, European Centre for International Political Economy: Policy Brief 3/2016. 
tätsrechte zu schützen. So waren insbesondere die Jahre, in denen Margaret Thatcher Premierministerin war, von harten Verhandlungen geprägt. Das wohl prominenteste Ergebnis ist der im Jahr 1984 ausgehandelte britische Rabatt auf die Beiträge des Landes zum Haushalt der Europäischen Union. Aktuellere Beispiele für den ausgeprägten Souveränitätsreflex Großbritanniens sind der „European Union Act“ von 2011,9 der ein Referendum erforderlich macht, sobald ein Souveränitätstransfer von der nationalen auf die europäische Ebene angestrebt wird, oder Camerons Veto zum Fiskalpakt im Dezember 2011.

Diese schwierigen Beziehungen zwischen Großbritannien und der Europäischen Union können mit dem Grundsatz, immer vom „besten beider Welten“ profitieren zu wollen (,getting the best of both worlds ${ }^{\text {“10 }}$ ), erklärt werden, der die britische Europapolitik prägt. Dies bedeutet, dass die politische Elite die Vorteile des europäischen Binnenmarkts klar anerkennt und auch danach strebt, die europäische Außenpolitik aktiv mitzugestalten. Gleichzeitig fehlt die Bereitschaft, sich in allen Bereichen vollkommen der europäischen Integration zu verschreiben. Dabei folgt die britische Europapolitik einem klaren KostenNutzen-Kalkül. Immer wieder wurden dementsprechend rote Linien nationaler Souveränität definiert und in Brüssel verteidigt. Großbritannien ist zum Beispiel das einzige Land der Europäischen Union, das offiziell fünf ökonomische Tests durchgeführt hat, um zu überprüfen, welchen Nutzen eine gemeinsame Währung für Großbritannien hätte und ob das Land bereit ist, dieser beizutreten. Auch Camerons Auftrag zur Überprüfung der Möglichkeiten und der Notwendigkeit zur Rückübertragung von Kompetenzen im Jahr 2013 entspricht dieser Logik britischer Europapolitik - auch wenn diese Tests ohne bemerkenswerte Ergebnisse geblieben sind.

Allgemein können neben dieser britischen Fokussierung auf den ökonomischen Nutzen europäischer Integration die Bevorzugung intergouvernementaler Kooperationsformen gegenüber supranationaler Politikgestaltung sowie das Werben für weitere EU-Erweiterungen mit dem Ziel, eine eher lose integrierte Europäische Union zu schaffen, als weitere Reibungspunkte zwischen Großbritannien und der Europäischen Union beziehungsweise den anderen EU-Mitgliedstaaten identifiziert werden. Diese Grundhaltungen haben immer wieder einen erheblichen Verhandlungsaufwand generiert - wie zum Beispiel beim bereits erwähnten britischen Rabatt oder der Einheitlichen Europäischen Akte in den 1980er Jahren, bei der Wirtschafts- und Währungsunion und dem Schengen-Raum in den 1990er Jahren und beim Vertrag über eine Verfassung für Europa oder dem Management der Finanzkrise seit 2008. Allerdings hat dies den europäischen Integrationsprozess bisher nicht aufhalten können, denn die Lösungen waren immer wieder Sonderregelungen und Opt-outRechte für Großbritannien.

\section{Gewinner oder Verlierer der differenzierten Integration in der Europäischen Union?}

Für eine umfassende Einschätzung der Auswirkungen möglicher Brexit-Szenarien ist es hilfreich zu rekapitulieren, welchen Platz Großbritannien aktuell in der Europäischen Uni-

9 European Union Act 2011: An Act to make provision about treaties relating to the European Union and decisions made under them, including provision implementing the Protocol signed at Brussels on 23 June 2010 amending the Protocol (No. 36) on transitional provisions annexed to the Treaty on European Union, to the Treaty on the Functioning of the European Union and to the Treaty establishing the European Atomic Energy Community; and to make provision about the means by which directly applicable or directly effective European Union law has effect in the United Kingdom, 19.7.2011, abrufbar unter: http://www.legislation.gov.uk/u kpga/2011/12/pdfs/ukpga_20110012_en.pdf (letzter Zugriff: 19.7.2016).

10 Siehe David Cameron: The Future of Britain's Relationship with the European Union. Rede bei Chatham House. London, 10. November 2015, abrufbar unter: https://www.chathamhouse.org/event/future-britains-rel ationship-european-union (letzter Zugriff: 19.7.2016). 
on - genauer gesagt in der differenzierten Europäischen Union - einnimmt. Wie Grafik 1 zeigt, hat kein anderes Land in der Union mehr Opt-outs von der europäischen Integration erreicht als Großbritannien.

\section{Grafik 1: Europa in Vielfalt geeint}

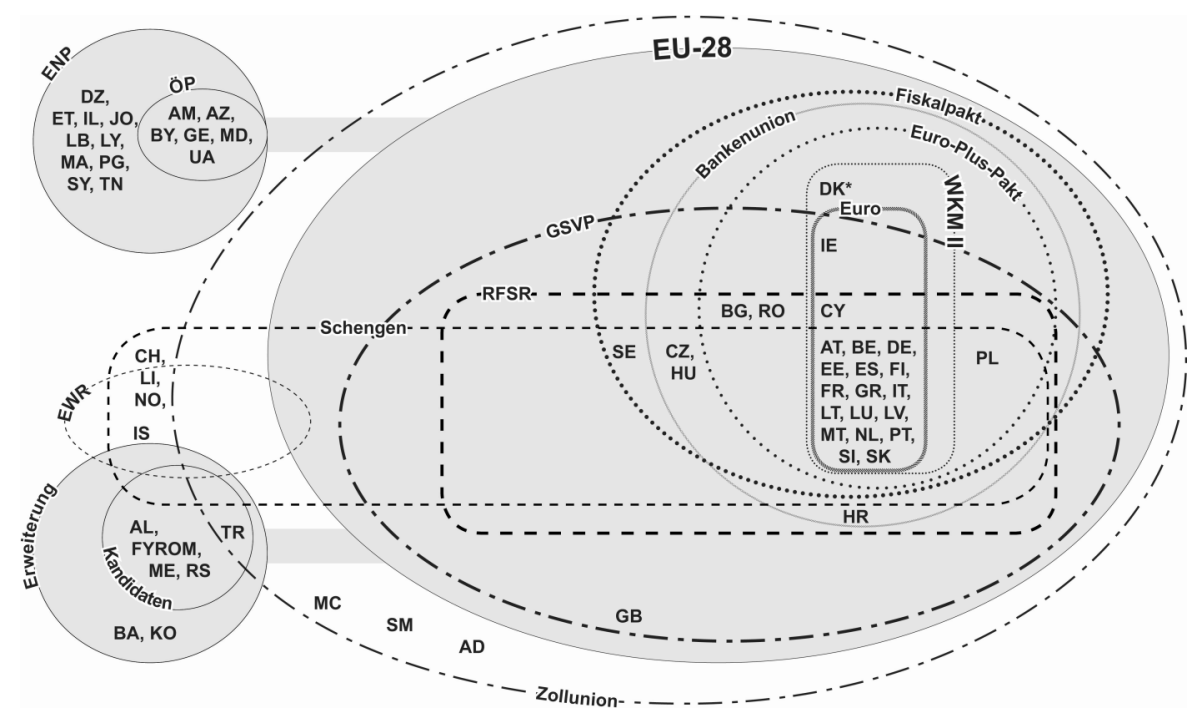

* Dänemark hat das Schengen-Abkommen unterzeichnet.

Abkürzung der Staaten: AD (Andorra); AL (Albanien); AM (Armenien); AT (Österreich); AZ (Aserbaidschan); BA (Bosnien-Herzegowina); BE (Belgien); BG (Bulgarien); BY (Weißrussland); CH (Schweiz); CY (Zypern); CZ (Tschechische Republik); DE (Deutschland); DK (Dänemark); DZ (Algerien); EE (Estland); EG (Ägypten); ES (Spanien); FI (Finnland); FR (Frankreich); FYROM (Ehemalige Jugoslawische Republik Mazedonien); GB (Vereinigtes Königreich); GE (Georgien); GR (Griechenland); HR (Kroatien); HU (Ungarn); IE (Irland); IL (Israel); IS (Island); IT (Italien); JO (Jordanien); KO (Kosovo); LB (Libanon); LI (Liechtenstein); LT (Litauen); LU (Luxemburg); LV (Lettland); LY (Libyen); MA (Marokko); MC (Monaco); MD (Moldawien); ME (Montenegro); MT (Malta); NL (Niederlande); NO (Norwegen); PG (Palästinensische Gebiete); PL (Polen); PT (Portugal); RO (Rumänien); RS (Serbien); SE (Schweden); SI (Slowenien); SK (Slowakei); SM (San Marino); SY (Syrien); TN (Tunesien); TR (Türkei); UA (Ukraine).

Sonstige Abkürzungen: ENP (Europäische Nachbarschaftspolitik); EWR (Europäischer Wirtschaftsraum); GSVP (Gemeinsame Sicherheits- und Verteidigungspolitik); ÖP (Östliche Partnerschaft); RFSR (Raum der Freiheit, der Sicherheit und des Rechts); WKM II (Wechselkursmechanismus II).

Quelle: Eigene Darstellung.

In der Wirtschafts- und Währungsunion hat Großbritannien genau wie Dänemark ein Opt-out von der Gemeinschaftswährung. Alle anderen EU-Mitgliedstaaten, die aktuell den Euro noch nicht eingeführt haben, sind verpflichtet, dies zu tun, sobald sie die Konvergenzkriterien erfüllen. Während in Dänemark die politische Elite bereits mehrfach ein Referendum zur Einführung des Euro in Erwägung gezogen hat, existiert in Großbritannien kein Interesse, der Eurozone beizutreten. Entsprechend nimmt Großbritannien anders als Dänemark auch nicht an den verschiedenen neuen Instrumenten zur Stärkung der Wirtschafts-, Fiskal- oder Bankenunion teil. In Anbetracht der verschiedenen Reformvorschläge zur zukünftigen Ausgestaltung der Europäischen Union zeichnet sich die Eurozone als 
ein mögliches ,Kerneuropa ${ }^{6} a b,{ }^{11}$ an dem Großbritannien allerdings nicht beteiligt ist (siehe auch Grafik 1).

Großbritannien genießt darüber hinaus Sonderrechte im Raum der Freiheit, der Sicherheit und des Rechts (RFSR) und im Schengen-Raum. Irland und Dänemark haben diesbezüglich ebenfalls, wenn auch weniger umfassende, Sonderstellungen. Weder Großbritannien noch Irland sind verpflichtet, am RFSR oder Schengen-Raum teilzunehmen (Opt-out). Beide Länder haben aber das Recht, ad hoc zu entscheiden, an welchen Einzelmaßnahmen dieser Politikfelder sie sich beteiligen möchten (Opt-in). ${ }^{12}$ Dänemarks Sonderrechte sind dahingehend anders strukturiert, dass es keine Opt-in-Rechte im Bereich des RFSR besitzt. Gleichzeitig ist Dänemark als Schengen-Unterzeichnerstaat aber verpflichtet, den Schengen-Besitzstand umzusetzen, ohne an der entsprechenden Gesetzgebung beteiligt zu sein. ${ }^{13}$ Dänemark könnte ein Opt-in-Regime wie Großbritannien und Irland einführen. Hierfür wäre allerdings die Zustimmung der Bevölkerung in einem Referendum notwendig. Ein solches scheiterte am 3. Dezember 2015. Somit wird Dänemark erst einmal ein Außenseiter in diesen Politikbereichen bleiben.

Dieses komplexe Regelwerk von Opt-out- und Opt-in-Rechten im RFSR und im Schengen-Raum entspricht dem britischen Grundsatz des ,getting the best of both worlds'. So hatte Großbritannien zum Beispiel die Möglichkeit, sich nicht an den umstrittenen Beschlüssen zur Umverteilung von insgesamt 160.000 Flüchtlingen aus Italien und Griechenland in andere Mitgliedstaaten im September 2015 zu beteiligen. Die Möglichkeiten Großbritanniens zum Rosinenpicken gingen sogar so weit, dass die britische Regierung im Jahr 2014 entscheiden konnte, sich aller Rechtsakte im Bereich der polizeilichen und justiziellen Zusammenarbeit in Strafsachen zu entledigen, die vor dem Inkrafttreten des Vertrags von Lissabon im Rahmen der undifferenzierten intergouvernementalen Gesetzgebung der sogenannten dritten Säule beschlossen worden waren. Dies wurde gemeinhin als ,BlockOpt-out' bekannt. ${ }^{14}$ Hintergrund hierfür war das Ende der Übergangsperiode zum 1. Dezember 2014, nach deren Ablauf der Gerichtshof der Europäischen Union die volle Jurisdiktion in diesen Rechtsbereichen erhielt. In einem zweiten Schritt konnten dann die Rechtsakte herausgesucht werden, die im Interesse Großbritanniens waren und daher weiterhin ihre Gültigkeit behalten sollten (Opt-in). Für diesen hohen Grad an Flexibilität bei der Beteiligung im RFSR und im Schengen-Raum zahlt Großbritannien allerdings den Preis der Einbußen bei den Möglichkeiten zur politischen Einflussnahme.

Eine besonders bemerkenswerte Sonderregelung für Großbritannien in der Europäischen Union ist nun durch die Entscheidung im Referendum für den Brexit erst einmal obsolet geworden. Die nach langen und zähen Verhandlungen am 18. und 19. Februar 2016 vom Europäischen Rat verabschiedeten neuen Regelungen für das Vereinigte Königreich innerhalb der Europäischen Union sahen vor, dass das Land ,in Anbetracht seiner

11 Zum Beispiel Glienicker Gruppe: Towards a Euro Union, 17.10.2013, abrufbar unter: http://glienickergruppe. eu/en/towards-a-euro-union/ (letzter Zugriff: 21.7.2016); The Eiffel Group: For a Euro Community, in: bruegel.org, 25.8.2014; The Spinelli Group/Bertelsmann Stiftung (Hrsg.): A Fundamental Law of the European Union, Gütersloh 2013.

12 Protokoll (Nr. 19) über den in den Rahmen der Europäischen Union einbezogenen Schengen-Besitzstand zum Vertrag von Lissabon, in: Amtsblatt der EU, Nr. C 83 vom 30. März 2010, S. 1-403, hier S. 290-292; und Protokoll (Nr. 21) über die Position des Vereinigten Königreichs und Irlands hinsichtlich des Raums der Freiheit, der Sicherheit und des Rechts zum Vertrag von Lissabon, in: ebenda, S. 295-298.

13 Protokoll (Nr. 22) über die Position Dänemarks zum Vertrag von Lissabon, in: ebenda, S. 299-303.

14 Funda Tekin: The UK's Block-Opt-Out - Serious Effects or Yet Another Peculiarity in EU-UK Relations?, Centre international de formation européenne: Policy Paper 6/2014. 
Sonderstellung nach Maßgabe der Verträge nicht zu einer weiteren politischen Integration in die Europäische Union verpflichtet ist" und somit vom Grundsatz der ,immer engeren Union“ offiziell entbunden ist. ${ }^{15}$ Allerdings sollten diese Regelungen nur dann in Kraft treten, wenn das Referendum für einen Verbleib Großbritanniens in der Europäischen Union ausgegangen wäre. Seit dem 23. Juni 2016 sind die Karten nun also wieder neu gemischt und die Beziehungen zwischen Großbritannien und der Europäischen Union müssen neu geregelt werden.

Dieser kurze Überblick über die britischen Sonderregelungen umfasst lediglich die als sogenannte Opt-outs im Primärrecht verankerten Differenzierungsmodalitäten. Auf die Darstellung der Flexibilisierungsregelungen im Sekundärrecht der Europäischen Union wird aus Gründen der Übersichtlichkeit verzichtet. Die hier präsentierten Sonderregeln sollen aber ausreichen, um Großbritannien als ,Meister ' der differenzierten Integration auszumachen. Allerdings bleibt festzustellen, dass diese ,Auszeichnung' den Preis der Marginalisierung innerhalb der Europäischen Union kostet. Dies zeigt sich besonders deutlich in der Wirtschafts- und Währungsunion. Hier wurden über die Jahre mit der EuroGruppe und dem Euro-Gipfel verstärkt eigene Entscheidungsgremien institutionalisiert, an denen Großbritannien nicht teilnimmt.

\section{Brexit ist nicht gleich Brexit: drei Szenarien möglicher Neugestaltung der Beziehungen zwischen Großbritannien und der Europäischen Union}

\section{Der konzeptionelle Rahmen: differenzierte Integration und differenzierte Desintegration}

Eine Vielzahl von Konzepten differenzierter Integration prägt die politische und akademische Debatte zum europäischen Integrationsprozess, wobei es des Öfteren an semantischer Klarheit fehlt. ${ }^{16}$ Das Phänomen der Desintegration oder differenzierten Desintegration wurde hingegen in der wissenschaftlichen Auseinandersetzung mit der europäischen Integration bisher weitestgehend ausgeklammert. ${ }^{17}$ Dies lässt sich zunächst ganz einfach dadurch erklären, dass Formen differenzierter Integration in den Jahrzehnten der Entwicklung der Europäischen Union und insbesondere während der sogenannten Eurozonenkrise der letzten Jahre deutlich zugenommen haben, während veritable Desintegrationstendenzen bislang noch ausgeblieben sind.

Eine umfassende Aufarbeitung der verschiedenen Konzepte differenzierter (Des-)Integration würde über das Ziel dieses Beitrags hinausgehen. ${ }^{18}$ Allerdings soll hier eine grundlegende Definition präsentiert werden, die die Analyse möglicher Konsequenzen verschiedener Brexit-Szenarien strukturiert. Diese lehnt sich an intergouvernementale beziehungsweise ,rational choice'-Ansätze europäischer Integrationstheorien an. Aus dieser Perspektive steigt in einer Europäischen Union mit 28 Mitgliedstaaten die Heterogenität der objektiven Fähigkeiten und politischen Interessen hinsichtlich Ausmaß und Inhalten des gemeinsamen Projekts. Eine entsprechende Kompromissfindung zwischen den Partikular-

15 Beschluss der im Europäischen Rat vereinigten Staats- und Regierungschefs über eine neue Regelung für das Vereinigte Königreich innerhalb der Europäischen Union, in: Europäischer Rat: Tagung des Europäischen Rates (18. und 19. Februar 2016). Schlussfolgerungen, EUCO 1/16, Anlage I-VII, S. 8-36, hier S. 16.

16 Für einen umfassenden Überblick siehe Alexander C-G. Stubb: A Categorization of Differentiated Integration, in: Journal of Common Market Studies 2/1996, S. 283-295.

17 Ausnahmen sind unter anderem Scheller/Eppler: European Disintegration, 2014; Webber: How likely is it that the European Union will disintegrate?, 2014.

18 Vgl. dazu Lisa H. Anders/Annegret Eppler/Thomas Tuntschew: Europäische Integration: zweidirektional und mehrdimensional, in diesem Band, S. 198-212. 
interessen einzelner EU-Mitgliedstaaten wird damit zu einer zentralen Herausforderung für den europäischen Integrationsprozess - insbesondere bei Vertragsreformen, die Einstimmigkeit sowie Ratifizierungen in allen Mitgliedstaaten entsprechend der Vorgaben ihrer jeweiligen Verfassung erfordern. ${ }^{19}$

Im Lichte dieser Überlegung lässt sich europäische Integration vereinfacht als ein Prozess von Vertiefung und Erweiterung verstehen, das heißt als ein Souveränitätstransfer auf die supranationale Ebene bei zunehmender Vergemeinschaftung der Entscheidungsmechanismen beziehungsweise als die territoriale Expansion der Europäischen Union. ${ }^{20}$ Die Desintegration konstituiert zunächst einmal den „Erosionsprozess“ einer solchen Integration, ${ }^{21}$ den Douglas Webber genauer definiert hat. Er identifiziert rückläufige Tendenzen in drei Bereichen als für dieses Phänomen charakteristisch: „(1) die Bandbreite an gemeinsamen Politiken, die auf EU-Ebene beschlossen werden, (2) die Anzahl an Mitgliedstaaten und (3) die formale Kapazität der EU-Institutionen, auch gegen den Willen einzelner Mitgliedstaaten Entscheidungen zu treffen und durchzusetzen."22

Differenzierte Integration kann als ein Instrument für das Heterogenitätsmanagement zwischen den EU-Mitgliedstaaten interpretiert werden, das dazu dient, diesen Erosionsprozess zu verhindern. Das grundlegende Prinzip ist, dass „eine Gruppe von Mitgliedstaaten nicht denselben Unionsregeln unterliegt wie die anderen“. ${ }^{23}$ Ein solcher Prozess ermöglicht „verschiedene Wege der Integration für verschiedene Mitgliedstaaten und gestatte $[\mathrm{t}]$ es denjenigen, die die Integration vertiefen möchten, weiter voranzugehen, wobei sie die Rechte derjenigen achten, die diesen Weg nicht einschlagen wollen".${ }^{24}$ Entsprechend dieser Definition ist differenzierte Integration immer dann politisch legitimiert, wenn sie der europäischen Integration dienlich ist und Opt-out-Rechte nur dann gewährt werden, wenn damit eine Blockade weiterer Integrationsschritte verhindert wird. Differenzierte Desintegration hingegen folgt der umgekehrten Logik, indem sie unterschiedliche Wege der Desintegration für verschiedene Mitgliedstaaten gestattet. Somit stellt dies den Prozess dar, bei dem EU-Mitgliedstaaten unterschiedlich weit die europäische Integrationsleiter wieder hinabsteigen würden. Ein Hauptunterscheidungsmerkmal ist daher, ob Differenzierung als Instrument für mehr oder weniger Integration dient.

Zwei Kriterien sollen hier für eine entsprechende Unterscheidung herangezogen werden. Zum einen ist es für den Integrationsfortschritt wichtig, dass Differenzierung offen strukturiert ist, sodass die Staaten, die zunächst nicht teilnehmen, zur vorangeschrittenen Gruppe aufschließen können, ohne dass letztere eine Veto-Option hätte. ${ }^{25}$ Differenzierung sollte demnach Flexibilität aufweisen, sodass die Möglichkeit gegeben ist, dass über kurz oder lang alle Mitgliedstaaten denselben Integrationsgrad erreichen. Dieses Prinzip ist am deutlichsten in der Differenzierung der ,mehreren Geschwindigkeiten“ umgesetzt. Aber auch Vertreter eines sogenannten Kerneuropas bemühen sich grundsätzlich zu betonen,

19 Art. 48 Vertrag über die Europäische Union (EUV).

20 Siehe Sara B. Hobolt: Ever closer or ever wider? Public attitudes towards further enlargement and integration in the European Union, in: Journal of European Public Policy 5/2014, S. 664-680; Anne Faber: Theoretical Approaches to EU Deepening and Widening: A Multi-disciplinary Overview and Some Tentative (Hypo)theses, EU-CONSENT: Paper, Oktober 2006.

21 Scheller/Eppler: European Disintegration, 2014, S. 24.

22 Webber: How likely is it that the European Union will disintegrate?, 2014, S. 342.

23 Funda Tekin/Wolfgang Wessels: Flexibility within the Lisbon Treaty: Trademark or Empty Promise?, in: Eipascope 1/2008, S. 25-31, hier S. 25.

24 Europäischer Rat: Schlussfolgerungen, Februar 2016, S. 9.

25 Thierry Chopin/Christian Lequesne: Differentiation as a double-edged sword: member states' practices and Brexit, in: International Affairs 3/2016, S. 531-545, hier S. 532. 
dass ein solcher Kern anderen EU-Mitgliedstaaten offenstehen sollte. ${ }^{26}$ Zum anderen kann Differenzierung unterschiedliche politische Impulse geben. Zunehmende Differenzierung kann eine Vertiefung im Integrationsprozess ermöglichen. Sie kann am Ende aber auch zu einem losen sogenannten Rosinenpicken führen, bei dem Staaten die Möglichkeit haben, interessengeleitet Kooperationen in einzelnen Politikfeldern ohne einen gemeinsamen europäisch-rechtlichen Rahmen einzugehen. Mitgliedstaatliche Einzelinteressen, die im Zweifel durch einen steigenden Europaskeptizismus geprägt sind, würden in diesem Fall den europäischen (Des-)Integrationsprozess dominieren. In der Geschichte der europäischen Integration waren es immer wieder britische Stimmen, die dieses ,l'Europe à la carte propagiert haben. ${ }^{27}$

Die Frage des Brexits erfordert eine weitere Unterscheidung der differenzierten Integration, die auch Frank Schimmelfennig, Dirk Leuffen und Berthold Rittberger vornehmen. Differenzierte Integration kann zwei Dimensionen beinhalten. Die interne Dimension bedeutet, dass EU-Mitgliedstaaten nicht an allen EU-Politiken beteiligt sind. Dies entspricht dem hier diskutierten Szenario (2) des ,Ins mit Opt-outs'. Externe differenzierte Integration bezieht sich hingegen auf Nicht-EU-Mitgliedstaaten, die an einer Auswahl von EUPolitiken teilnehmen - die sogenannten, Outs mit Opt-ins ${ }^{6}{ }^{28}$

Aus diesen Überlegungen heraus ergibt sich ein Dreischritt für die Analyse der möglichen Brexit-Szenarien. Die drei oben genannten Szenarien werden zunächst anhand prozessualer und struktureller Merkmale sowie anhand von Effekten für die Europäische Union und Großbritannien unterschieden. In einem zweiten Schritt werden sie in den Kontext aktueller Integrationsstrategien und -visionen gesetzt, um das Ausmaß und die Flexibilität möglicher Differenzierung zu bemessen. Zusätzlich sollen die Szenarien auf die von ihnen ausgehende Gefahr eines Dominoeffekts auf andere Mitgliedstaaten überprüft werden. Dies ermöglicht die Bewertung hinsichtlich der Wahrscheinlichkeit eines, l'Europe à la carte'.

\section{Die politischen Rahmenbedingungen: Brisanz und Risiken eines Brexits}

Die Entscheidung der britischen Bevölkerung für einen Austritt Großbritanniens aus der Europäischen Union wäre auch in Zeiten stabiler europäischer Integration als historisch zu bezeichnen gewesen. Folgende aktuelle Faktoren erhöhen darüber hinaus ihre Brisanz sowie mögliche Risiken.

Die Europäische Union hat in ihrer Geschichte eine Vielzahl an Krisen durchlaufen ${ }^{29}$ und ist dem allgemeinen Verständnis nach daran gewachsen. Aktuell befindet sich die Europäische Union jedoch in einer „Polikrise“.30 Seit 2008 kämpft die Eurozone mit den Konsequenzen der internationalen Finanzkrise. Im Sommer 2015 konnte zwar das Aus-

26 Zum Beispiel Glienicker Gruppe: Towards a Euro Union, 2013; The Eiffel Group: For a Euro Community, 2014; The Spinelli Group/Bertelsmann Stiftung: A Fundamental Law of the European Union, 2013.

27 Zuletzt David Cameron: EU speech at Bloomberg. London, 23. Januar 2013, abrufbar unter: https://www.gov .uk/government/speeches/eu-speech-at-bloomberg (letzter Zugriff: 21.7.2016).

28 Frank Schimmelfennig/Dirk Leuffen/Berthold Rittberger: The European Union as a System of Differentiated Integration: Interdependence, Politicization and Differentiation, Institute for Advanced Studies: Political Science Series Working Paper 137/2014.

29 Hartmut Kaelble: Spirale nach unten oder produktive Krisen? Zur Geschichte politischer Entscheidungskrisen der europäischen Integration, in: integration 3/2013, S. 169-182.

30 Europäisches Parlament: Draft Report on possible evolutions of and adjustments to the current institutional set-up of the European Union, Committee on Constitutional Affairs, Berichterstatter: Guy Verhofstadt, 5.7.2016, 2014/2248(INI), S. 5 . 
scheiden Griechenlands aus der Gemeinschaftswährung - der sogenannte Grexit - in letzter Minute abgewendet werden. Aber Griechenlands Wirtschaft ist weit davon entfernt gesundet zu sein. Gegen Portugal und Spanien wurde darüber hinaus im Juli 2016 zum ersten Mal das Defizitsanktionsverfahren eingeleitet. Obwohl immer noch virulent, sind diese Instabilitäten in der Eurozone Ende 2015 und Anfang 2016 fast gänzlich aus dem Fokus der politischen und öffentlichen Debatte gerückt. Grund hierfür ist der exponentielle Anstieg der Migrationszahlen aufgrund der Krisen im Nahen Osten und Nordafrika. Alleine im Jahr 2015 wurden mehr als 1,3 Millionen Flüchtlinge in der Europäischen Union registriert. Davon reichten 476.510 (knapp 37 Prozent) einen Antrag auf Asyl in Deutschland ein. ${ }^{31}$ Hieraus manifestierte sich eine Krise, die den Schengen-Raum unter extremen Druck stellte. Obwohl die Flüchtlingszahlen seit dem ersten Quartal 2016 aufgrund der Schließung der Balkanroute sowie eines Abkommens mit der Türkei erheblich zurückgegangen sind, ist diese Krise noch nicht überwunden. Gründe hierfür sind unter anderem die schwierigen türkisch-europäischen Beziehungen sowie die innenpolitische Instabilität nach dem missglückten Putschversuch in der Türkei vom 15. Juli 2016.

Diese ,Polikrise' wirkt sich auf das von Andreas Hofmann und Wolfgang Wessels definierte Ebenendilemma aus, welches die EU-Mitgliedstaaten bei der Gestaltung der europäischen Integration lösen müssen. Ein solches Dilemma resultiert daraus, dass in einem Mehrebenensystem die Ebene gefunden werden muss, die zur jeweils effektivsten und effizientesten Politiklösung führt. Zum einen erkennen die Mitgliedstaaten an, dass für einige grenzüberschreitende Politikbereiche die optimale Problemlösungsebene die europäische ist. Dies gilt insbesondere für den Schengen-Raum und die damit verbundenen Politiken wie die Migrationspolitik oder die polizeiliche und justizielle Zusammenarbeit in Strafsachen. ${ }^{32}$ Der Souveränitätsreflex konterkariert diesen Problemlösungsinstinkt aus Sorge vor der Aushöhlung der De-jure-Souveränität des Nationalstaates. ${ }^{33}$ Diese Sorge dominiert krisenbedingt die aktuelle Europapolitik, sodass nationalstaatliche Partikularinteressen im Vordergrund stehen.

Ergänzt und unterstützt wird dieser aktuelle Trend durch einen starken Zulauf bei europaskeptischen Parteien und Bewegungen, nicht nur in den traditionell für solche Tendenzen bekannten Mitgliedstaaten wie Dänemark, Großbritannien, die Niederlande oder Polen, sondern auch in Frankreich und nicht zuletzt Deutschland. Eine aktuelle Studie des Pew Research Centers zeigt, dass im Durchschnitt lediglich 51 Prozent der Bürger die Eu-

31 Die offiziell erhobenen Daten zu der genauen Anzahl an Asylbewerbern variieren. Die hier genannten stammen von Eurostat und beinhalten einen geringen Anteil an Zweitanträgen. Die circa 820.000 Asylbewerber, die im Jahr 2015 keinen Antrag in Deutschland eingereicht haben, suchten Asyl in den restlichen 27 EU-Mitgliedstaaten. Hierbei liegen die Zahlen der Asylbewerber in den einzelnen Ländern weit unter 100.000. Lediglich Ungarn (177.130) und Schweden (162.455) stellen eine Ausnahme dar. Siehe Eurostat: Asylbewerber und erstmalige Asylbewerber nach Staatsangehörigkeit, Alter und Geschlecht Monatliche Daten (gerundet), Stand 23.3.2016, abrufbar unter: http://ec.europa.eu/eurostat/web/products-datasets/-/migr_asyappctzm (letzter Zugriff: 30.3.2016).

32 Siehe hierzu auch Funda Tekin: Europapolitische Prioritäten Deutschlands in den Bereichen Innere Sicherheit, Asyl- und Einwanderungspolitik, in: Mathias Jopp/Katrin Böttger (Hrsg.): Handbuch zur deutschen Europapolitik, Baden-Baden 2016, S. 341-356.

33 Andreas Hofmann/Wolfgang Wessels: Eine dauerhafte Verfassung für Europa? Die Beantwortung konstitutioneller Grundfragen durch den Vertrag von Lissabon, in: Frank Decker/Marcus Höreth (Hrsg.): Die Verfassung Europas. Perspektiven eines Integrationsprojekts, Wiesbaden 2009, S. 69-95. 
ropäische Union als etwas Positives betrachten. 42 Prozent fordern eine Rückverlagerung von Kompetenzen. ${ }^{34}$

Diese Dominanz des Souveränitätsreflexes und der Europaskeptizismus stellen keinen Nährboden für positive Effekte differenzierter Integration dar. Das heißt, sie erzeugen ein Klima, in dem Differenzierungstendenzen den europäischen Integrationsprozess nicht unbedingt fördern, sondern im Gegensatz durchaus auch Desintegrationspotenzial aufweisen.

Ein Brexit könnte demzufolge riskante Konsequenzen für die europäische Integration haben. Das Risiko wird noch durch eine fehlende „Austrittsdoktrin“ der Europäischen Union $^{35}$ verstärkt. Bisher existiert lediglich das in Art. 50 EUV festgeschriebene Recht, aus der Europäischen Union auszutreten. Dieser Vertragsartikel definiert das Verfahren des Austritts. Der genaue Gegenstand des Austrittsabkommens wird hingegen offengelassen. Dasselbe gilt für den Zeitpunkt der Absichtserklärung, die Großbritannien an den Europäischen Rat zu richten hat. ${ }^{36}$ Hieraus können sich politische Gestaltungsspielräume ergeben. So wurde zum Beispiel in den Tagen direkt nach dem Referendum darüber spekuliert, ob Großbritannien am Ende tatsächlich ein Austrittsgesuch an den Europäischen Rat stellen wird. Die oben zitierte Aussage von May bei ihrem Amtsantritt als britische Premierministerin, „Brexit heißt Brexit“, die sie bei ihrem Besuch bei Bundeskanzlerin Angela Merkel am 20. Juli 2016 wiederholte, ${ }^{37}$ hat diesen Spekulationen zumindest vorerst ein Ende bereitet. Es scheint auch klar, dass Großbritannien erst Anfang 2017 das sogenannte Artikel-50-Verfahren einleiten wird. Bis dahin bliebe also genügend Zeit, den Versuch zu starten, auf informellem Wege verschiedene Optionen auszuloten und mögliche Verbündete unter den verbleibenden 27 EU-Mitgliedstaaten zu mobilisieren. Letztere wollen jedoch größtmögliche Geschlossenheit wahren und Verhandlungen idealerweise nur im Rahmen des formellen Verfahrens führen.

Zum gegenwärtigen Zeitpunkt haben weder Großbritannien noch die Europäische Union klare Forderungen hinsichtlich der Neuordnung ihrer Beziehungen gestellt. Die hier diskutierten Szenarien orientieren sich daher an den rechtlichen und strukturellen Möglichkeiten eines Brexits. Darüber hinaus sollen aktuell diskutierte Integrationsstrategien rahmengebend sein. Das Ergebnis des Referendums wurde von den politischen Vertretern in Europa auch als Aufforderung verstanden, den europäischen Integrationsprozess zu überdenken. In diesem Beitrag sollen daher die Überlegungen der Außenminister Deutschlands und Frankreichs, des Präsidenten des Europäischen Parlaments, Martin Schulz, sowie des Ausschusses für konstitutionelle Fragen des Europäischen Parlaments im sogenannten Verhofstadt-Bericht Beachtung finden. ${ }^{38}$ Obwohl diese Gedankenspiele unter-

34 Dieser Durchschnittswert bezieht sich auf empirische Erhebungen in den zehn EU-Mitgliedstaaten Deutschland, Frankreich, Griechenland, Großbritannien, Italien, Niederlande, Polen, Schweden, Spanien und Ungarn. Siehe Bruce Stokes: Euroskepticism Beyond Brexit. Significant opposition in key European countries to an ever closer EU, Pew Research Center, 7. Juni 2016.

35 Barbara Lippert/Nicolai von Ondarza: Der Brexit als Neuland. Mit dem britischen Referendum beginnt ein komplexer Austrittsprozess - und vielleicht die Erneuerung der EU, Stiftung Wissenschaft und Politik: SWPAktuell 42/2016.

36 Art. 50 Abs. 2 EUV.

37 Die Bundesregierung: Pressekonferenz von Bundeskanzlerin Merkel und der britischen Premierministerin May. Berlin, 20. Juli 2016, abrufbar unter: https://www.bundesregierung.de/Content/DE/Mitschrift/Pressekon ferenzen/2016/07/2016-07-20-merkel-may.html (letzter Zugriff: 21.7.2016).

38 Siehe Frank-Walter Steinmeier/Jean-Marc Ayrault: Ein starkes Europa in einer unsicheren Welt. Gemeinsamer Beitrag. Berlin, 27. Juni 2016, abrufbar unter: https://www.auswaertiges-amt.de/DE/Europa/Aktuell/160 624-BM-AM-FRA_ST.html (letzter Zugriff: 21.7.2016); Martin Schulz: Mit Herzblut und Leidenschaft, in: Frankfurter Allgemeine Zeitung, 11.7.2016; Europäisches Parlament: Draft Report, 2016. 
schiedliche Ausprägungen hauptsächlich hinsichtlich der demokratischen Neugestaltung der Europäischen Union sowie des Zeitplans für mögliche Reformen aufweisen, so stimmen sie doch in zwei Punkten überein. Erstens: Die Funktionsfähigkeit der Europäischen Union muss verbessert werden. Das bedeutet weder ein schnelles „,mehr Europa“ ${ }^{\text {“39 oder }}$ ein einfaches „weiter so“40 noch eine „bloße Reflexionsphase“",41 wichtig sei hingegen, Probleme zu identifizieren und für diese konkrete und klare Lösungen anzubieten. Zweitens: In allen Überlegungen wird differenzierte Integration als eines der hauptsächlichen Charakteristika zukünftiger europäischer Integrationsschritte anerkannt. Frank-Walter Steinmeier und Jean-Marc Ayrault sprechen von ,unterschiedlichen Ambitionsniveaus“ 42 der EU-Mitgliedstaaten, denen Rechnung getragen werden müsse. Guy Verhofstadt kritisiert die aktuelle Ausgestaltung der differenzierten Integration beziehungsweise ,variablen Geometrien“" in der Europäischen Union, bei der die Mitgliedstaaten vermehrt den ,à la carte-Ansatz" verfolgen würden. Er fordert die Reduktion der Komplexität von differenzierter Integration. Eine mögliche Lösung sieht er darin, Grundelemente einer EU-Mitgliedschaft zu definieren und Staaten, die einer solchen Mitgliedschaft nicht uneingeschränkt zustimmen, einen Assoziierungsstatus zu gewähren. ${ }^{43}$

\section{Vollständiger Austritt Großbritanniens aus der Europäischen Union - ein unintendiertes Szenario?}

Rein formal betrachtet besteht die Möglichkeit, dass Großbritannien vollständig aus der Europäischen Union austritt. Allerdings handelt es sich hierbei um die zweitgrößte Volkswirtschaft in der Europäischen Union, die wirtschaftlich eng mit dem europäischen Kontinent verbunden ist. Mehr als 50 Prozent britischer Importe kommen aus der Europäischen Union und mehr als 50 Prozent der britischen Exporte gehen in die EU-Staaten. ${ }^{44}$ Auch in außenpolitischen Fragen sind beide Seiten relevant füreinander. Im Falle eines Brexits würde die Europäische Union unter anderem Einfluss im Sicherheitsrat der Vereinten Nationen einbüßen, wo Großbritannien einen ständigen Sitz innehat. Unter Berücksichtigung dieser Aspekte dürften sowohl die Europäische Union als auch Großbritannien ein eher geringes Interesse an einem vollständigen Austritt haben.

Allerdings könnte es unter Umständen zu einem sogenannten ungeordneten (kompletten) Austritt kommen. Art. 50 Abs. 3 EUV sieht nach der Einreichung der Absichtserklärung eine Periode von zwei Jahren für die Verhandlungen des Austrittsabkommens vor. Sollte zu diesem Zeitpunkt kein Verhandlungsergebnis vorliegen, verlieren die Verträge ihre Anwendung auf Großbritannien. Der Europäische Rat kann allerdings im Einvernehmen mit Großbritannien einstimmig beschließen, einen solchen unvorhergesehenen Austritt zu verhindern und die Frist zu verlängern.

Solche Szenarien eines kompletten Austritts wären wohl aus politischer und auch wirtschaftlicher Sicht sowohl für Großbritannien als auch für die Europäische Union die unattraktivsten. Allerdings hätten sie innerhalb der Europäischen Union das geringste Differenzierungs- und Desintegrationspotenzial zur Folge. Ferner dürften in diesem Szenario die Nachteile eines Austritts und die Vorteile der EU-Mitgliedschaft besonders deutlich

39 Steinmeier/Ayrault: Ein starkes Europa, 2016, S. 1.

40 Schulz: Mit Herzblut und Leidenschaft, 2016.

41 Steinmeier/Ayrault: Ein starkes Europa, 2016, S. 1.

42 Ebenda, S. 2.

43 Europäisches Parlament: Draft Report, 2016, S. 7.

44 Siehe hierzu auch Iain Begg: Brexit: warum, was nun und wie?, in diesem Band, S. 230-241. 
werden. Dies dürfte daher den Nachahmungseffekt bei anderen Staaten in Grenzen halten. Bereits kurz nach dem Referendum in Großbritannien und den Aussagen politischer Vertreter der EU-Mitgliedstaaten, einen Brexit hart verhandeln zu wollen, zeichnete sich ab, dass ein Rückgang der Befürwortung ähnlicher Referenden in anderen europaskeptischen Mitgliedstaaten zu erwarten ist. In Dänemark, wo traditionell ein starker Europaskeptizismus die Europapolitik prägt, ${ }^{45}$ wurde die Forderung nach einem Referendum sehr schnell leiser - der Zuspruch hierfür sank von 41 auf 32 Prozent. Gleichzeitig war ein Anstieg bei der Unterstützung für die dänische EU-Mitgliedschaft auf 69 Prozent zu verzeichnen. ${ }^{46}$ Auch in anderen Ländern wie der Tschechischen Republik oder Frankreich, wo starke europaskeptische Tendenzen erkennbar sind, zielen die Forderungen eher auf Reformen der Europäischen Union als auf einen Austritt ab. ${ }^{47}$ Allerdings dürften Reformvorstellungen dort von Schulz' Plänen für einen Ausbau der europäischen Institutionen zu einer Europäischen Regierung ${ }^{48}$ abweichen und eher auf intergouvernementalere Strukturen zielen. Es wäre also falsch anzunehmen, dass ein Brexit automatisch eine Gelegenheit zur grundlegenden Umstrukturierung und stärkeren Vertiefung der Europäischen Union darstellt, nur weil einer der politisch gewichtigsten Integrationsgegner ausgetreten ist. Der aktuell propagierte Ansatz, konkrete Antworten auf Teilprobleme der europäischen Integration zu suchen, scheint die bessere Strategie, um Differenzierungs- und Desintegrationstendenzen vorzubeugen.

Das Szenario eines vollständigen Austritts hätte allerdings Potenzial, Desintegrationsbeziehungsweise Sezessionstendenzen in Großbritannien auszulösen. Erst im Jahr 2014 hatte Schottland in einem Referendum die Zugehörigkeit zum Vereinigten Königreich bestätigt. Ein wichtiger Beweggrund hierfür war der Wunsch, die Europäische Union nicht verlassen zu müssen. Im EU-Referendum am 23. Juni 2016 stimmten 62 Prozent der Schotten gegen einen Brexit. Die schottische ,Frage“ bedarf daher im Falle eines vollständigen Brexits einer Lösung - im Zweifel sogar einer Sonderlösung, denn ein Austritt lediglich eines Teils eines EU-Mitgliedstaats ist in den Verträgen nicht vorgesehen. Daher kann Schottland zum Zeitpunkt eines Brexits nicht einfach EU-Mitglied bleiben. Es müsste in einem erneuten Referendum für die Unabhängigkeit stimmen, um selbständig einen Antrag auf EU-Mitgliedschaft stellen zu können. Abgesehen davon, dass ein zweites schottisches Referendum für nicht sehr wahrscheinlich gehalten wird, ${ }^{49}$ müsste Schottland auch bilateral Lösungen mit der Europäischen Union verhandeln.

Ein vollständiger Brexit würde zumindest für die Europäische Union trotz des Verlustes eines Mitgliedstaates am wenigsten Veränderung bedeuten. Auch wenn es ohne Großbritannien schwierig sein dürfte, eine veritable europäische Regierung in naher Zukunft zu etablieren, so ist eine umfassende Repatriierung der Kompetenzen von europäischer Ebene und somit ein Rückgang in der Bandbreite gemeinsamer Politiken in diesem Szenario nicht zu erwarten.

45 Siehe auch Informationen zum Opt-out oben.

46 Gabriel Samuels: EU membership support surges in Denmark after Brexit vote, in: The Independent, 5.7.2016.

47 Stokes: Euroskepticism Beyond Brexit, 2016.

48 Schulz: Mit Herzblut und Leidenschaft, 2016.

49 Siehe Begg: Brexit, in diesem Band. 


\section{Austritt Großbritanniens aus der Europäischen Union mit Assoziierung - das externe Differenzierungsszenario eines Outs mit Opt-ins}

Am prominentesten werden aktuell mögliche Szenarien eines Brexits mit anschließender Assoziierung Großbritanniens an die Europäische Union diskutiert. Dies würde bedeuten, dass im Rahmen von Art. 50 EUV ein Austrittsabkommen beschlossen und daraufhin eine Form der Assoziierung verhandelt wird. Aufgrund des Fehlens einer rechtlich definierten ,assoziierten Mitgliedschaft", die Andrew Duff, ehemaliges Mitglied des Europäischen Parlaments für die britischen Liberal Democrats, 2013 vorgeschlagen hat ${ }^{50}$ und die auch im Verhofstadt-Bericht als Idee wieder aufgenommen wird, ${ }^{51}$ sind unterschiedliche Modelle der Anbindung Großbritanniens an die Europäische Union vorstellbar. ${ }^{52}$

Das sogenannte Norwegen-Modell würde bedeuten, Großbritannien tritt dem Europäischen Wirtschaftsraum (EWR) bei und verbleibt dadurch im Binnenmarkt. Allerdings müsste Großbritannien in diesem Fall einiges akzeptieren, was im Vorfeld des Referendums als inakzeptabel deklariert worden war: EWR-Mitglieder übernehmen den Acquis communautaire im Bereich des Binnenmarktes, der einen Großteil des gesamten EUBesitzstands ausmacht, ohne an den entsprechenden Entscheidungen beteiligt zu sein; sie müssen entsprechend auch in den EU-Haushalt einzahlen; und eine Teilnahme am europäischen Binnenmarkt setzt voraus, dass alle vier Grundfreiheiten vollständig gewährleistet werden. Großbritannien wehrt sich jedoch gegen die Personenfreizügigkeit, die die Arbeitsmigration im Binnenmarkt ermöglicht. Aus diesem Grund werden auch ,EWRMinus'-Szenarien diskutiert, die es Großbritannien basierend auf Art. 112 und 113 des EWR-Vertrags $^{53}$ ermöglichen würden, Arbeitsmigration zu limitieren. Dennoch würde Großbritannien sich in stark asymmetrische Beziehungen mit der Europäischen Union begeben. Diese hat ihrerseits bereits verkündet, dass eine Teilnahme Großbritanniens am Binnenmarkt ,à la carte' nicht verhandelbar sei. ${ }^{54}$ Die Freizügigkeiten stellen die Eckpfeiler des Binnenmarktes dar. Die Gewährung eines Opt-outs für Großbritannien würde nicht nur gegen das Nichtdiskriminierungsprinzip verstoßen, sondern könnte andere EU-Mitgliedstaaten dazu animieren, dasselbe zu fordern. Ein Binnenmarkt ,à la carte' würde daher ein substanzielles Desintegrationspotenzial aufweisen. Die Form der externen differenzierten Integration über den Weg der Assoziierung Großbritanniens als Nicht-EU-Mitglied könnte somit interne differenzierte Desintegrationstendenzen auslösen.

Das sogenannte Schweizer Modell, bei dem Großbritannien eine Vielzahl von bilateralen Assoziierungsverträgen mit der Europäischen Union aushandelt, würde größere Flexibilität und Nutzen für Großbritannien bedeuten. Gleichzeitig wäre der Verhandlungsaufwand nach den Erfahrungen mit der Schweiz erheblich, sodass es höchst unwahrscheinlich ist, dass sich die Europäische Union darauf einlassen wird oder sollte.

Aufgrund der Tatsache, dass diese Modelle bedeuten, dass Großbritannien zwar wirtschaftlich weiter vom europäischen Binnenmarkt profitieren, gleichzeitig jedoch keinerlei

50 Andrew Duff: The case for an Associate Membership of the European Union, in: EUROPP Blog, 3.6.2013.

51 Europäisches Parlament: Draft Report, 2016.

52 Siehe hierzu Nicolai von Ondarza: Zwischen den Welten. Großbritannien als Partner für Deutschland in Europa nach dem EU-Referendum, Stiftung Wissenschaft und Politik: SWP-Aktuell 35/2016; Pol Morillas (Hrsg.): The Brexit Scenarios: Towards a New UK-EU Relationship, Barcelona Centre for International Affairs: CIDOB Documents 7/2016.

53 Abkommen über den Europäischen Wirtschaftsraum, in: Amtsblatt der EG, Nr. L 1 vom 3. Januar 1994, S. 3-522.

54 Europäischer Rat, Der Präsident: Ausführungen von Präsident Donald Tusk nach der informellen Tagung der Staats- und Regierungschefs aus 27 Mitgliedstaaten der EU. Brüssel, 29. Juni 2016, Doc. 396/16. 
politischen Einfluss mehr hierauf nehmen könnte, erscheinen auch diese Szenarien eher unattraktiv für andere EU-Mitgliedstaaten. Das Ausmaß eines möglichen Dominoeffekts wird dabei allerdings auch davon abhängen, wie strikt die Europäische Union ihre Verhandlungsposition durchsetzen wird. Das heißt, je mehr die hier dargestellten Modelle aufgeweicht werden, desto attraktiver könnten diese für potenzielle Nachahmer werden. Auch aus schottischer Sicht wird die weitere Strategie davon abhängen, welche Rechte und Pflichten für Großbritannien am Ende bestehen bleiben.

\section{Kein Austritt Großbritanniens aus der Europäischen Union, sondern Verbleib mit mehr Sonderrechten - das interne Differenzierungsszenario des Ins mit Opt-outs}

Trotz der wiederholten Versicherung durch die neue britische Premierministerin, dass das Ergebnis des Referendums ernst genommen und ein Austrittsgesuch an den Europäischen Rat übermittelt werden wird, sollte das Szenario, bei dem der Brexit nicht stattfindet, mit in die Analyse einbezogen werden. Zum einen könnte es sein, dass das Artikel-50Verfahren entgegen aller Beteuerungen am Ende doch nicht lanciert wird. Hierauf setzen momentan zumindest die britischen Buchmacher. ${ }^{55}$ Eine Variante besteht darin, dass das Austrittsgesuch während der Verhandlungen wieder zurückgenommen wird. Dies ist gemäß Art. 50 EUV zumindest nicht verboten. In diesem Fall hätte Großbritannien unter extremen Bedingungen für beide Seiten Camerons Mission fortgeführt und die Mitgliedschaft in der Europäischen Union nochmals neu verhandelt.

Szenario drei würde einen extremen internen Differenzierungsschub in der Europäischen Union zur Folge haben. Zum einen würde Großbritannien weitere Opt-outs verlangen - auch wenn, wie oben bereits dargelegt, die Grundfreiheiten des europäischen Binnenmarkts hier ebenfalls nicht verhandelbar sein dürften. Großbritannien würde weiterhin eine Ausnahme von dem Grundprinzip einer ,immer engeren Union` einfordern. Da dies bereits Gegenstand der von Cameron erreichten Neuregelung für das Vereinigte Königreich in der Europäischen Union war, dürften die anderen EU-Mitgliedstaaten wieder zu diesem Zugeständnis bereit sein. Zugleich ist die Gefahr eines Dominoeffekts bei den anderen EU-Mitgliedstaaten in diesem Szenario am größten. Großbritannien hat über die Jahrzehnte europäischer Integration wiederholt Sonderregelungen erhalten. Der Brexit als Druckmittel würde diese Verhandlungen ins Extrem führen und andere Mitgliedstaaten könnten in Versuchung geraten, ihre Partikularinteressen auf gleichem Wege durchzusetzen. Das Risiko eines veritablen ,l'Europe à la carte' mit umfassender Rückverlagerung von Kompetenzen sowie einer Zunahme an intergouvernemental ausgestalteten Politiken wäre also hoch. Ein für Partikularinteressen besonders ,attraktiver' Politikbereich ist die europäische Migrationspolitik. Ungarn plant ein Referendum über die Umsetzung der EUBeschlüsse zur Umverteilung von insgesamt 160.000 Asylsuchenden. Diese Entscheidung wurde gegen den Willen Ungarns und der Slowakei mit qualifizierter Mehrheit im Rat durchgesetzt. Bei zunehmender Gewährung von Sonderrechten für Großbritannien könnte die Umsetzung von EU-Recht in Zukunft noch schwieriger werden, weil die Solidarität unter den Mitgliedstaaten zunehmend schwindet.

Im Falle eines Verbleibs Großbritanniens in der Europäischen Union mit zusätzlichen Sonderrechten sollte daher über eine grundlegende Reform der Europäischen Union sowie der differenzierten Integration nachgedacht werden. Der Vorschlag von Verhofstadt eines

55 Matthew Nitch Smith: Brexit betting: Bookmakers are more sure than ever that Article 50 will never be triggered, in: Business Insider Deutschland, 20.7.2016. 
inneren Zirkels von Ländern mit einer EU-Vollmitgliedschaft und Peripheriestaaten stellt in dieser Hinsicht einen Anfang dar. Was diesem Reformvorschlag jedoch fehlt, ist eine klare Definition von Mechanismen, die es einem Staat der Peripherie ermöglichen, in den Kern zu wechseln. Somit scheint dieses Konzept zwar differenzierte Integration klarer zu strukturieren, gleichzeitig jedoch auch zu verfestigen. Zusätzlich sollten die Vorschläge zur Demokratisierung der Europäischen Union und auch zu Lösungen für Teilprobleme in der Migrations-, Außen- und Sicherheits- sowie Wirtschafts- und Währungspolitik weiter ausgearbeitet werden. Letzteres würde darauf abzielen, den Vertrauensverlust in die Europäische Union auszugleichen und abzubauen, und würde damit zumindest eine Grundlage für einen möglichen Rückgang europaskeptischer Bewegungen in Europa bilden.

\section{Zusammenfassung und Ausblick: Brexit als Katalysator für differenzierte (Des-)Integration}

Nachdem das erste Erstaunen über den Ausgang des britischen EU-Referendums und die damit einhergegangenen Befürchtungen über dessen Folgen für die Zukunft der Europäischen Union überwunden sind, hat dieser Beitrag gezeigt, dass weder das Referendum noch ein tatsächlicher Brexit das Ende europäischer Integration bedeuten. Zugleich sind wegweisende Konsequenzen für den zukünftigen Integrationsprozess zu erwarten. Die Verhandlungen über einen Brexit sowie ein tatsächlicher Austritt Großbritanniens aus der Europäischen Union werden nicht nur die Debatte über differenzierte Integration wieder neu beleben, sondern diese auch als ein Strukturmerkmal der Europäischen Union verfestigen. Echte Desintegrationstendenzen hingegen sind nur dann zu befürchten, wenn Großbritannien zu viele Zugeständnisse gemacht werden. Die Grundfreiheiten des Binnenmarktes sollten dabei eine rote Linie darstellen, da sie den Punkt markieren, an dem sich differenzierte Integration in differenzierte Desintegration wandelt. 